\title{
REVIEW
}

\section{A comprehensive analysis of smart home energy management system optimization techniques}

\author{
Tesfahun Molla ${ }^{1}$, Baseem Khan ${ }^{*}$, Pawan Singh ${ }^{2}$ \\ ${ }^{1}$ School of Electrical \& Computer Engineering, Hawassa University Institute of Technology, Hawassa, 05, Ethiopia \\ ${ }^{2}$ School of Informatics, Hawassa University Institute of Technology, Hawassa, 05, Ethiopia
}

\begin{abstract}
Development of smart grid technology provides an opportunity to various consumers in context for scheduling their energy utilization pattern by themselves. The main aim of this whole exercise is to minimize energy utilization and reduce the peak to average ratio (PAR) of power. The two way flow of information between electric utilities and consumers in smart grid opened new areas of applications. The main component is this management system is energy management controller (EMC), which collects demand response (DR) i.e. real time energy price from various appliances through the home gateway (HG). An optimum energy scheduling pattern is achieved by EMC through the utilization of DR information. This optimum energy schedule is provided to various appliances via HG. The rooftop photovoltaic system used as local generation micro grid in the home and can be integrated to the national grid. Under such energy management scheme, whenever solar generation is more than the home appliances energy demand, extra power is supplied back to the grid. Consequently, different appliances in consumer premises run in the most efficient way in terms of money. Therefore this work provides the comprehensive review of different smart home appliances optimization techniques, which are based on mathematical and heuristic one.
\end{abstract}

Keywords: home appliances energy management system; heuristic techniques; mathematical techniques; energy management system

ARTICLE INFO

Received: March 20, 2018

Accepted: April 10, 2018

Available online: May 15, 2018

*CORRESPONDING AUTHOR Baseem Khan, School of Electrical \& Computer Engineering, Hawassa University Institute of Technology, Hawassa, 05, Ethiopia;

baseem.khan04@gmail.com

\section{CITATION}

Molla T, Khan B and Singh P. A comprehensive analysis of smart home energy management system optimization techniques. Journal of Autonomous Intelligence 2018; 1(1)

\section{Introduction}

Due to the ever growing demand of electrical energy, the percentage of renewable energy generation is greatly raised along with the increased in energy prices. Various environmental constraints also posed a limit on energy generation from conventional energy sources ${ }^{[1]}$. These all the challenges motivate power industry to shift their focus on smart demand side management techniques. According to ${ }^{[2]}$, out of $100 \%$ of energy utilized in USA by residential and commercial consumers, at least $30 \%$ energy is wasted. The advancement of information and communication technologies (ICTs) increased the demand of reliable and quality power supply ${ }^{[3]}$. These ICTs are important part of smart grid system that transfers information from one system to another one. This information transfer is very helpful to control and coordinate various smart grid technologies to respond immediately under varying demand situation. Smart grids can use local renewable energies like wind and solar energy to solve environmental Problems, increase reliability of equipment and system and reduce the costs of infrastructure ${ }^{[4]}$. Further to control the demand from consumer premises, these ICT systems are very helpful. These are the integral part of smart home appliances energy management system, which is a one aspect of smart grid $^{[5]}$.

Today, the concept of micro-grid is being used for purpose of helping the environment through using several renewable and available resources such as wind and solar energy, along with other energy generators such as microturbines and fuel cells to reduce generation costs and reduce environmental pollutions. The resources are not only interrelated, but also the interaction continues in higher levels such as distribution networks. Restriction of fossil fuels and increasing growth of demand for energy, enhanced living standards, 
global warming and environmental problems have led to increasing advancements in technology and use of modern energies. The idea of smart grid was begun with idea of Advanced Metering Infrastructure (AMI) to develop demand management, increase energy efficiency and a self-repair electric grid, so that it could improve reliability and responding to natural disasters or deliberate sabotage ${ }^{[4]}$. Advancement of smart grid system allows consumers to reduce their energy consumption through proper scheduling of different appliances. This is possible with the information of real time electricity pricing (RTEP). The demand side management (DSM) techniques are very old in power system that incorporates DR techniques with load shifting and energy efficiency and conservation program. Shifting of consumers' loads from peak hours to off peak hours is the main function of DR techniques. For that purpose incentives are offered to consumers ${ }^{[6]}$.

DR programs transfer customers' load during periods of high-demand to off-peak periods by offering them incentives and can reduce critical peak demand or daily peak demand ${ }^{[6]}$. In smart grid environment there are different DR pricing schemes. The most common pricing schemes include real-time (RTP), time of use (TOUP) and critical peak (CPP).

In this paper, authors present a comprehensive study of optimization techniques of smart home energy management system. In Section 2, the architecture of smart home energy management system is studied under a home area network (HAN) protocol. In section 3, authors reviewed HEMS optimization methods based on mathematical and heuristic techniques. Section 4 presents a comparative analysis between different methods followed by the conclusion

\section{The structure of home energy management system in smart grid}

It is assumed that a smart home consists of

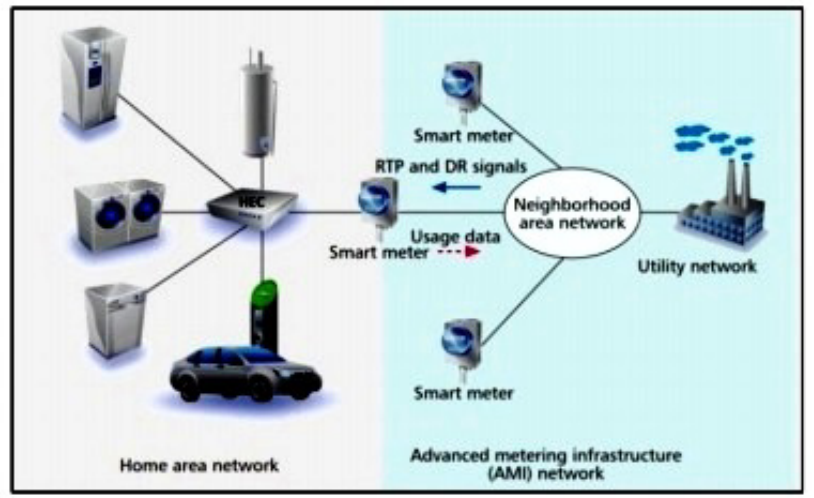

Figure 1. Home energy management system architecture $^{[1]}$ various appliances having the adequate and proper communication interfaces in order to facilitate the exchange of information with the EMS. It also assumed that each of the appliances communicates with the EMS only and cannot communicate with each other.

Figure 1 presents the structure of home energy management system with different data communication domains, those domains includes the internet domain, home area network (HAN) domain and the smart meter domain (AMI). The internet domain enables consumers to monitor and control their power consumption profile, the scheduling of their appliance power consumption and etc. through an in-home display (IHD) which consists of computer, tablet or smart phone. The smart meter domain includes a large number of interconnected smart meters called "Automatic metering infrastructure (AMI)" which is installed and monitored by utilities companies in order to transmit load information and DR signals between the smart homes and power market. Based on RTP signals received 24 hours day ahead, the home energy management system (EMS) schedules the electricity usage pattern for the next day.

\section{Literature Review}

\subsection{Mathematical Techniques}

\subsubsection{Mix Integer Linear Programming}

Arikiez et $a l .{ }^{[7]}$ investigated the use of linear programming based heuristics for solving the cost minimization problem of air conditioning system for residential and commercial loads. Melhem et $a l .{ }^{[8]}$ proposed an optimal residential load scheduling model by using the mixed integer linear programming. Sou et al. ${ }^{[9]}$ solved scheduling problem of smart home appliances for reducing electricity cost by using mixed integer linear programming. Angelis et al. ${ }^{[10]}$ developed an approach, which is based on mixed-integer linear programming technique. The developed approach provided an optimal solution in terms of minimum power consumption with an integration of renewable energy resources. Yao et al. ${ }^{[11]}$ investigated a problem of a smart home energy management system (HEMS). It consist of photovoltaic (PV) module and the household appliances with three different types of load characteristics (i.e., interruptible, uninterruptible and time-varying). To solve the HEMS problem mixed integer linear programming 
(MILP) algorithm was used. Kim et al. ${ }^{[12]}$ developed a mixed-integer linear programming based model for an effective scheduling of the operational periods and power levels of home appliances. Arora et al. ${ }^{[13]}$. Proposed a mixed integer linear programming based optimal scheduling technique for centralized air conditioning load. Same author proposed an optimal scheduling mechanism for residential appliances in a smart home equipped with photovoltaic panel and energy storage device ${ }^{[14]}$. In order to reduce both the $\mathrm{CO} 2$ emissions and the electricity bill, Paridari et $a l .{ }^{[15]}$ proposed a novel based on MILP method for smart home appliances scheduling and batteries, Same author ${ }^{[16]}$ proposed a robust approach based on MILP model for scheduling of smart home appliances by incorporating electrical energy storage system (ESS) in active buildings with the aim of minimizing both the $\mathrm{CO} 2$ emissions and electricity billing. Zavala et al. ${ }^{[17]}$ developed a proactive energy management framework based on online solution of mixed-integer nonlinear programming. It is an integrated predictive dynamic home models and day-ahead forecasting of disturbances which affects the efficiency of the system and electricity costs. In order to jointly monitor the electricity consumption of home appliances and plug-in EVs, Hossain et $a l .{ }^{[18]}$ proposed an optimal centralized scheduling method, which is based on MILP. For an efficient management of smart home appliances under real time pricing (RTP) environment, Wang et al. ${ }^{[19]}$ proposed a Mixed Integer Linear Programming (MILP) based rolling optimization. The proposed smart home equipped with a Battery Energy Storage System (BESS) and a solar PV system in order to maximize the efficiency of the system.

\subsubsection{Linear Programming}

Giorgio et al.$^{[20]}$ proposed the design of a Smart Home Controller which provides an efficient management of electric energy in a residential building. To solve the problem, binary linear programming scheme was used. Dogaheh et $a l .{ }^{[2]}$ optimized the performance of smart grids in relation with residential energy centres equipping with solar power units or PV. For that purpose authors utilised YALMIP toolbox in MATLAB software. Mohammadi et al. ${ }^{[22]}$ proposed a real time integer linear programming based Home Energy Management system (HEMS), to manage home appliances and storage system.

\subsection{Heuristic Techniques}

\subsubsection{Particle Swarm Optimization PSO}

Hussain et al. ${ }^{[23]}$ proposed a particle swarm optimization (PSO) based scheduler using demand side management (DSM) technique based on demand response (DR). Six shiftable appliances are considered in 24 hour time horizon. PAR is not included as objective function. Shah et al. ${ }^{[24]}$, presented a HEMS using multi-agent system (MAS) for smart homes. The HEMS uses priority techniques with the integration of electrical supply system (ESS). Further, bio-inspired technique, binary particle swarm optimization (BPSO), is used for the optimal scheduling of appliances in a smart home. Mahmood et al. ${ }^{[25]}$, proposed a realistic scheduling mechanism (RSM) to reduce user frustration and enhance appliance utility by classifying appliances with respective constraints and their time of use effectively. Further, binary particle swarm optimization (BPSO) is utilized for optimal scheduling of appliances with constraints. Huang et al. ${ }^{[26]}$ studied a convex optimization problem to manage the energy consumption of different households in an automatic way. In addition to this optimal scheduling is performed by hybrid PSO-DE algorithm. Zhu et al. ${ }^{[27]}$ utilized cooperative PSO algorithm for developing the optimal schedule and control of energy and time shiftable appliances in smart homes. Abdalla et al ${ }^{[28]}$ studied the energy management of chiller plant in optimal way. PSO and Fuzzy are utilized for optimally managing the energy consumption of plant. To find the set point, fuzzy is utilized while for optimizing the objective function PSO is used. For plug-in hybrid electric vehicles, Chen et al. ${ }^{[29]}$ developed an optimum energy management method under uncertain driving conditions. Authors utilized PSO for optimizing the threshold parameters for the rule based energy management scheme.

\subsubsection{Gray Wolf Optimization}

Kazemi et al.$^{[30]}$ developed a method that depends on Gray wolf optimization and genetic algorithm to achieve the optimal schedule for appliances in terms of cost and PAR. Interruptible tasks are divided in to smaller sub tasks, in terms of time, using GWO algorithm and the output of this component was the input of genetic algorithm scheduler (GAS). The inclining block rate (IBR) pricing model was proposed to restrict the overusing electricity power 
in each time slice. Even if it takes large time to converge to the solution, the performance of the optimization technique seems interesting and had a good result than other methods discussed above.

\subsubsection{Genetic algorithm GA}

Zhao et al. ${ }^{[31]}$ introduced an EMS in HAN based smart grid. Further, GA is utilized to solve the problem of energy scheduling for home appliances. An EMS and automated DR structure for household is developed by Khomami et al. ${ }^{[32]}$ to schedule the different appliance in smart household. Further, for optimizing the results GA is utilized by the authors. For optimal DR management in smart grid Meng et al. $^{[33]}$ developed a real time pricing technique. A Stackelberg Game and Genetic Algorithm Approach are utilized for that purpose. Talha et al. ${ }^{[34]}$ evaluated the performance of heuristic Algorithms: GA and Artificial Fish Swarm Algorithm (AFSA) for Demand Side Management. Li et al. ${ }^{[35]}$ developed an EMS for industrial microgrids with high renewable energy penetration. The scheme is developed for both isolated and grid connected mode with day ahead operation. For optimizing the results, regrouping particle swarm optimization (RegPSO) is utilized by the authors. To reduce the PAR and energy cost, Naseem et al ${ }^{[36]}$ scheduled the residential loads by four different heuristic optimization techniques i.e. GA, Binary Particle Swarm Optimization (BPSO), wind driven optimization (WDO) and Bacterial Forging Optimization Algorithm (BFOA). A comparative analysis of all the above mentioned techniques is presented by the authors.

\subsubsection{Evolutionary algorithm}

For future smart grid with large devices, Logenthiran et al. ${ }^{[37]}$ developed a DSM scheme that depends on load shifting. Evolutionary Algorithm (EA) is utilized for optimizing the results. Allerding et al. ${ }^{[38]}$ focused on a flexible EMS for Smart households. A "plug-and-play" type Evolutionary Algorithm is developed for optimizing distributed generation.

\subsubsection{Bee colony}

Zhang et al. ${ }^{[39]}$ proposed an EMS for household appliances. It is also utilized to implement DR schemes for residential consumers and for facilitating integration of renewable energy sources. For optimizing the results an improved artificial bee colony algorithm is used.

\subsubsection{Bacterial colony}

An internet of things (IOT) based EMS for households are proposed by Geng et al. ${ }^{[40]}$. For finding an optimum scheduling scheme, discrete multi-objective bacterial colony chemotaxis algorithm (DMOBCC) is utilized by the authors. Lin et al. ${ }^{[41]}$ presented an EMS for micro grid system. For optimizing the results of economic dispatch, enhanced bee colony optimization (EBCO) is developed by the authors.

\subsubsection{Ant colony}

Rahim et al. ${ }^{[42]}$ developed an optimization model for DSM that combined tariff and block

Table 1. Comparative analysis of Mathematical and Heuristic based methods

\begin{tabular}{|c|c|c|c|}
\hline $\begin{array}{l}\text { S. } \\
\text { No. }\end{array}$ & Properties & Mathematical Technique & Heuristic Techniques \\
\hline 1. & Model Formulation & $\begin{array}{l}\text { Model is used to simulate a system when } \\
\text { the system is not extremely complicated. }\end{array}$ & $\begin{array}{l}\text { Meta-heuristic Techniques try to } \\
\text { imitate natural phenomenon to solve } \\
\text { the complex practical problems. }\end{array}$ \\
\hline 2. & Prerequisites & $\begin{array}{l}\text { Physical laws are derived and utilized } \\
\text { to find the relationships between system } \\
\text { parameters. }\end{array}$ & $\begin{array}{l}\text { Dependent on data to determine the } \\
\text { structure and parameters }\end{array}$ \\
\hline 3. & $\begin{array}{l}\text { Non-linearity } \\
\text { integration }\end{array}$ & $\begin{array}{l}\text { Possible if prior knowledge of the nature of } \\
\text { the non-linearity exists. }\end{array}$ & $\begin{array}{l}\text { The prior information of the nature of } \\
\text { the non-linearity is not required. }\end{array}$ \\
\hline 4. & Hypothesis & $\begin{array}{l}\text { Simplify the problem or incorporating } \\
\text { assumptions to make model more } \\
\text { understandable. }\end{array}$ & $\begin{array}{l}\text { No need to simplify the model and/or } \\
\text { add assumptions }\end{array}$ \\
\hline 5. & Precision \& Accuracy & Provide highest level of accuracy. & $\begin{array}{l}\text { Provide greater precision and } \\
\text { accuracy. }\end{array}$ \\
\hline 6. & Efficiency & Time consuming in complex problem & $\begin{array}{l}\text { repetitive and time-consuming tasks } \\
\text { easily carried out with efficiency }\end{array}$ \\
\hline 7. & $\begin{array}{l}\text { Renewable Energy } \\
\text { Integration }\end{array}$ & Requires complex mathematical modelling & $\begin{array}{l}\text { With meta-heuristic model renewable } \\
\text { energy, source incorporation is easy. }\end{array}$ \\
\hline
\end{tabular}


rates. Multiple knapsacks are utilized to form the optimization problem and ant colony optimization is utilized to solve it.

\section{Comparative analysis of various optimization techniques}

In this section, a comparative analysis of different home energy management optimization methods is presented, which are reviewed in previous sections. Table 1 presents a comparison between mathematical and heuristic techniques based smart home energy management optimization methods. This comparison is based on different factors such as model formulation, prerequisites, nonlinearity integration, hypothesis, precision and accuracy, efficiency and renewable energy integration. From the presented comparison it is clear that both techniques has various advantages and limitations. Based on the formulated problem one can select suitable technique.

\section{Conclusion}

The problem for smart home appliance management is developed as a mathematical MILP and liner programming (LP)) or heuristic optimization problem with the decision variable. The number of appliances can be increased to add more types of appliances in a home area network (HAN). Optimized appliance scheduling can be studied with the other existing dynamic pricing models. Smart home energy management is becoming more important in smart grid environment. It is capable to deal with demand response and management techniques. The main objective of home energy management problem is to optimize the schedule of various home appliances so that the overall energy consumption minimised. Hence, in this work authors presents a comprehensive review of solution techniques and methods used for optimization of smart home energy management system. Different heuristic techniques used for optimization of smart HEMS are further discussed. At last, a comparative analysis among different smart home energy management techniques is presented. There are still many constraints and non-linearity, which should be incorporated in future smart home energy management problem. Problems related to mathematical validation, deregulated market constraints, contingencies incorporation, renewable sources integration are latest challenges for future smart home energy management problems.

\section{References}

1. Hadis Khomami, Mohammad Javidi. An Efficient Home Energy Management System for Automated Residential Demand Response, Environment and Electrical Engineering (EEEIC), 2013 13th International Conference on IEEE.

2. L. Geng, Z. Lu. A Novel Model for Home Energy Management System Based on Internet of Things, 2016 IEEE International Conference on Power and Renewable Energy.

3. Z. Zhao, W. Lee, Y. Shin, et al. An Optimal Power Scheduling Method for Demand Response in Home Energy Management System, IEEE Trans. on Smart Grid (Volume: 4, Issue: 3, Sept. 2013)

4. Z. Dogaheh, M. Dogaheh. Optimizing the Performance of Smart Grids in Relation with Residential Energy Centers Equipping with Solar Power Units (PV). Sindhological Studies, 2017(1): 14-22

5. S. Mohammadi, M. Momtazpou, E. Sanaei. Optimization-based Home Energy Management in the Presence of Solar Energy and Storage, IEEE, 2013.

6. F. A. Qayyum, M. Naeem, A. S. Khwaja, et al. Appliance Scheduling Optimization in Smart Home Networks, special section on smart grids, IEEE, October 29, 2015.

7. M. Arikiez, F. Grasso, M. Zito. Heuristics for the cost-effective management of a temperature controlled environment. 2015 IEEE Innovative Smart Grid TechnologiesAsia (ISGT ASIA), Bangkok, 2015: 1-6.

8. F. Y. Melhem, O. Grunder, Z. Hammoudan, et al. Optimal residential load scheduling model in smart grid environment, 2017 IEEE International Conference on Environment and Electrical Engineering and 2017 IEEE Industrial and Commercial Power Systems Europe (EEEIC / I\&CPS Europe), Milan, 2017: 1-6.

9. Kin Cheong Sou, James Weimer, Henrik Sandberg, et al. Scheduling Smart Home Appliances Using Mixed Integer Linear Programming. 50th IEEE Conference on Decision and Control and European Control Conference(CDC-ECC) Orlando, FL, USA, December 12-15, 2011.

10. F. De Angelis, M. Boaro, D. Fuselli, et al. Optimal Home Energy Management Under Dynamic Electrical and Thermal Constraints. IEEE Transactions on Industrial Informatics, 9(3): 1518-1527, August 2013. 
11. L. Yao, Z. Damiran, W. H. Lim. Energy management optimization scheme for smart home considering different types of appliances. 2017 IEEE International Conference on Environment and Electrical Engineering and 2017 IEEE Industrial and Commercial Power Systems Europe (EEEIC / I\&CPS Europe), Milan, 2017: 1-6.

12. Seung-Jun Kim, G. B. Giannakis. Efficient and scalable demand response for the smart power grid. 2011 4th IEEE International Workshop on Computational Advances in Multi-Sensor Adaptive Processing (CAMSAP), San Juan, 2011: 109-112.

13. M. Arora, S. Chanana, A. Kumar. A real time price based optimal scheduling mechanism for centralized air conditioning load. 2014 Eighteenth National Power Systems Conference (NPSC), Guwahati, 2014: 1-5.

14. M. Arora, S. Chanana. Residential demand response from PV panel and energy storage device. 2014 IEEE 6th India International Conference on Power Electronics (IICPE), Kurukshetra, 2014: 1-6.

15. Kaveh Paridari, Alessandra Parisio, Henrik Sandberg, et al. Energy and $\mathrm{CO} 2$ efficient scheduling of smart appliances in active houses equipped with batteries. 2014 IEEE International Conference on Automation Science and Engineering (CASE) Taipei, Taiwan, August 18-22, 2014.

16. K. Paridari, A. Parisio, H. Sandberg, et al. Robust Scheduling of Smart Appliances in Active Apartments With User Behavior Uncertainty. IEEE Transactions on Automation Science and Engineering, 13(1): 247-259, January 2016.

17. Victor M. Zavala, Jianhui Wang, Sven Leyffer. Proactive energy management for nextgeneration building systems. Fourth National Conference of IBPSA-USA New York City, New York, August 11-13, 2010.

18. M. H. K. Tushar, C. Assi, M. Maier, M. F. Uddin. Smart Micro grids: Optimal Joint Scheduling for Electric Vehicles and Home Appliances. IEEE Transactions on Smart Grid, 5(1): 239-250, January 2014.

19. H. Wang, K. Meng, Z. Y. Dong, et al. Efficient real-time residential energy management through MILP based rolling horizon optimization. 2015 IEEE Power \& Energy Society General Meeting, Denver, CO, 2015: $1-6$.

20. Alessandro Di Giorgio, Laura Pimpinella, Alessandra Quaresima, et al. An event driven Smart Home Controller enabling cost effective use of electric energy and automated Demand Side Management. 19th Mediterranean Conference on Control and Automation Aquis Corfu Holiday Palace, Corfu, Greece June 20-23, 2011

21. Zahra Rasouli Dogaheh, Mostafa Rasouli Dogaheh. Optimizing the Performance of Smart Grids in Relation with Residential Energy Centers Equipping with Solar Power Units (PV). Sindhological Studies, 2017(1): 14-22.

22. S. Mohammadi, M. Momtazpour, E. Sanaei. Optimization-based home energy management in the presence of solar energy and storage. 201321 st Iranian Conference on Electrical Engineering (ICEE), Mashhad, 2013: 1-6.

23. B. Hussain, Qadeer-U1-Hassan. Demand side management for smart homes in Pakistan. 2016 International Conference on Emerging Technologies (ICET), Islamabad, 2016: 1-6.

24. S. Shah, R. Khalid, A. Zafar, et al. An Optimized Priority Enabled Energy Management System for Smart Homes. 2017 IEEE 31st International Conference on Advanced Information Networking and Applications (AINA), Taipei, 2017: 10351041.

25. Danish Mahmood, Nadeem Javaid, Nabil Alrajeh, et al. Realistic Scheduling Mechanism for Smart Homes. Energies 2016, 9(3): 202.

26. Yantai Huang, Lei Wang, Qidi Wu. A Hybrid PSO-DE Algorithm for Smart Home Energy Management. ICSI 2014, Part II, LNCS 8795, 2014: 292-300.

27. Jiawei Zhu, Fabrice Lauri, Abderrafiaa Koukam, et al. Scheduling Optimization of Smart Homes Based on Demand Response. AIAI 2015, IFIP AICT 458, 2015: 223-236.

28. E. A. H. Abdalla, P. Nallagownden, N. M. Nor, et al. Intelligent approach for optimal energy management of chiller plant using fuzzy and PSO techniques. 2016 6th International Conference on Intelligent and Advanced Systems (ICIAS), Kuala Lumpur, 2016: 1-6.

29. Zeyu Chen, Rui Xiong, Jiayi Cao. Particle swarm optimization-based optimal power management of plug-in hybrid electric vehicles considering uncertain driving conditions. Energy 2016, 96(1): 197-208.

30. S. F. Kazemi, S. A. Motamedi, S. Sharifian. A home energy management system using Gray Wolf Optimizer in smart grids. 2017 2nd Conference on Swarm Intelligence and Evolutionary Computation (CSIEC), Kerman, 
2017: 159-164.

31. Z. Zhao, W. C. Lee, Y. Shin, et al. An Optimal Power Scheduling Method for Demand Response in Home Energy Management System. IEEE Transactions on Smart Grid 2013, 4(3): 1391-1400.

32. Hadis Pourasghar Khomami, M. H. Javidi. An efficient home energy management system for automated residential demand response. 2013 13th International Conference on Environment and Electrical Engineering (EEEIC), Wroclaw, 2013: 307-312.

33. Fan-Lin Meng, Xiao-Jun Zeng. An Optimal Real-time Pricing for Demandside Management: A Stackelberg Game and Genetic Algorithm Approach. 2014 International Joint Conference on Neural Networks (IJCNN) July 6-11, 2014, Beijing, China.

34. Muhammad Talha, Muhammad Shahid Saeed, Ghulam Mohiuddin, et al. Energy Optimization in Home Energy Management System Using Artificial Fish Swarm Algorithm and Genetic Algorithm. Advances in Intelligent Networking and Collaborative Systems, Lecture Notes on Data Engineering and Communications Technologies 8 .

35. Han Li1, Abinet Tesfaye Eseye, Jianhua Zhang, et al. Optimal energy management for industrial micro grids with high-penetration renewables. Protection and Control of Modern Power Systems 2017, 2:12.

36. Mudassar Naseem, Samia Abid, Rabia Khalid, et al. Towards Heuristic Algorithms: GA, WDO, BPSO, and BFOA for Home Energy Management in Smart Grid. Advances on Broad-Band Wireless Computing, Communication and Applications, Lecture Notes on Data Engineering and Communications Technologies 2.

37. T. Logenthiran, D. Srinivasan, T. Z. Shun. Demand Side Management in Smart Grid Using Heuristic Optimization. IEEE Transactions on Smart Grid 2012, 3(3): 12441252.

38. Florian Allerding, Ingo Mauser, Hartmut Schmeck. Customizable Energy Management in Smart Buildings Using Evolutionary Algorithms. EvoApplications 2014, LNCS 8602, 2014: 153-164.

39. Yanyu Zhang, Peng Zeng, Chuanzhi Zang. Optimization Algorithm for Home Energy Management System Based on Artificial Bee Colony in Smart Grid. The 5th Annual IEEE International Conference on Cyber Technology in Automation, Control and Intelligent Systems June 8-12, 2015, Shenyang, China.

40. Lijun Geng, Yi Wei, Zhigang Lu, et al. A novel model for home energy management system based on Internet of Things. 2016 IEEE International Conference on Power and Renewable Energy (ICPRE), Shanghai, 2016: 474-480.

41. Whei-Min Lin, Chia-Sheng Tu, Ming-Tang Tsai. Energy Management Strategy for Microgrids by Using Enhanced Bee Colony Optimization. Energies 2016, 9: 5.

42. S. Rahim et al. Ant Colony Optimization Based Energy Management Controller for Smart Grid. 2016 IEEE 30th International Conference on Advanced Information Networking and Applications (AINA), CransMontana, 2016: 1154-1159. 